\title{
Direction of Arrival Estimation for Speech Sources using Fourth Order Cross Cumulants
}

\author{
Mikael Swartling, Benny Sällberg, Nedelko Grbić \\ Department of Signal Processing, School of Engineering, Blekinge Institute of Technology \\ Box 520 SE-37225 Ronneby, Sweden
}

\begin{abstract}
In many applications where speech separation and enhancement is of interest, e.g. conferencing systems, mobile phones and hearing aids, accurate speaker localization is important. This paper presents an alternative criteria for the well known Steered Response Power with Phase Transform (SRPPHAT) algorithm, in which the steered response relates to peaks in the fourth order cross cumulant, rather than peaks in the second order cross cumulant, i.e. the cross power spectrum. Since speech sources have a Probability Density Function (PDF) close to the Laplacian distribution and noise are generally closer to the Gaussian distribution, the fourth order cumulant becomes a good alternative for the steered response search for speech sources. The proposed method is evaluated and compared to the original SRP-PHAT algorithm and shows significant improvements in localization performance for speech sources.
\end{abstract}

\section{INTRODUCTION}

In many applications, an accurate estimate of the location of an acoustic speech source is desired. An example of where speech source localization can be used is video conferencing systems with automatic speaker tracking of the camera. For reliable camera tracking, an accurate estimate of the speakers location is needed. In speech enhancement, accurate estimates may also be necessary, as in [1] where Time Difference of Arrival (TDOA) estimates are used to focus a beamformer towards an active speaker. Speech enhancement systems targets for example hands free sets for cars, mobile devices, laptop computers and conference telephony systems. A popular method lately for Direction of Arrival (DOA) estimation is the SRP-PHAT [2] which uses second order statistics through the use of cross power spectra to steer a beamformer, searching for a maxima in the power output from the beamformer. A peak in the power output means the beamformer is aimed towards the acoustic source with the highest power.

The use of fourth order statistics (the kurtosis measure) can provide a way to distinguish speech from noise. A Gaussian random process exhibit a cumulant with a zero value for any cumulant order higher than two, so estimation of higher order cumulants will in theory eliminate Gaussian noise [3]. Although not entirely Gaussian, most real noise sources have a probability density function close to the Gaussian distribution, whereas speech can be modeled more accurately as a Laplacian distribution [4]. This a-priori knowledge can be exploited in order to separate and enhance speech sources by for example maximizing the kurtosis measure of the output of a beamformer [5].
In this paper, a new method for localizing speech sources is presented. The method is based on the SRP-PHAT algorithm where a new optimization target is proposed. Instead of focusing on second order statistics (the cross power spectrum), fourth order statistics are used. The rationale behind choosing fourth order statistics when estimating DOA for speech sources being that speech sources exhibit larger fourth order cumulant values than most common noise sources compared to second order statistics that relates only to the power of the sources, and not specifically on their PDFs.

The outline of the paper is as follows. In section II the signal model and a brief description of the Generalized Cross Correlation (GCC), SRP and PHAT is presented, and in section III the fourth order cumulant is described. Section IV describes the proposed method for TDOA estimation. In section $\mathrm{V}$ the proposed method is evaluated and compared to the original SRP-PHAT algorithm, followed by a brief analysis of computational complexity. Conclusions are given in section VI.

\section{Delay Estimation Methods}

\section{A. Generalized Cross Correlation (GCC)}

Assuming the relative attenuation of a source between the sensors within the sensor array is negligible, the received signals at sensor $m \in[1,2, \ldots M]$ can be modeled as

$$
x_{m}(t)=s\left(t-\tau_{m}\right)+n_{m}(t)
$$

where $s(t)$ is the acoustic source signal, $\tau_{m}$ is the propagation delay from the source to sensor $m$ and $n_{m}(t)$ models the noise received by the $m$ :th sensor. The associated TDOA for sensor pair $\{m, n\}$ is defined as $\tau_{m, n}=\tau_{n}-\tau_{m}$. A summary of methods to estimate $\tau_{m, n}$ is presented in [2].

The method proposed in this paper is, at its very origin, based on the GCC method [6]. The GCC method for estimating TDOA can in its basic form be stated as

$$
\hat{\tau}_{m, n}=\arg \max _{\tau} R_{m, n}(\tau)
$$

where $\hat{\tau}_{m, n}$ is the estimated TDOA and $R_{m, n}(\tau)$ is the cross correlation between sensor signals $x_{m}(t)$ and $x_{n}(t)$. In a more general form, the weighted GCC can also be defined in frequency domain as

$$
\hat{\tau}_{m, n}=\arg \max _{\tau} \int_{-\infty}^{\infty} \psi(\Omega) G_{m, n}(\Omega) e^{j \Omega \tau} d \Omega
$$


where $\psi(\Omega)$ is the weighting function, $G_{m, n}(\Omega)$ is the cross power spectrum defined as

$$
G_{m, n}(\Omega)=E\left[X_{m}(\Omega) X_{n}^{*}(\Omega)\right]
$$

where $E[\cdot]$ is the expectation operator, $X_{m}(\Omega)$ and $X_{n}(\Omega)$ are frequency domain representations of $x_{m}(t)$ and $x_{n}(t)$, respectively, and $(\cdot)^{*}$ denotes complex conjugation.

A number of weighting functions are presented in [6]. One of the more popular weighting functions is the phase transform, defined as

$$
\psi_{\operatorname{PHAT}}(\Omega)=\frac{1}{\left|G_{m, n}(\Omega)\right|} .
$$

The phase transform has been shown to perform well for speech signals in reverberant environments [2].

\section{B. Steered Response Power (SRP)}

A further development of GCC is the SRP which extends the GCC to an arbitrary number of sensors and arbitrary sensor configurations. SRP type of algorithms are methods based on steering a beamformer across the search space in order to find a maxima in the response power in the beamformer output signal. The SRP of a filter-and-sum beamformer with $M$ sensors in a uniformly distributed linear sensor array can be stated as

$$
P(\tau)=\sum_{\{m, n\} \in \mathbf{P}_{-\infty}} \int_{-\infty}^{\infty} \psi(\Omega) G_{m, n}(\Omega) e^{j \Omega \tau(n-m)} d \Omega
$$

where $\mathbf{P}$ is the set of all combinations of sensor pairs within the array. The TDOA for an acoustic source is estimated as

$$
\hat{\tau}=\arg \max _{\tau} P(\tau) .
$$

The defined SRP is equivalent to the sum of the GCC for all combinations of sensor pairs in the sensor array, which means that for a two-sensor array the SRP is equivalent to the GCC. The SRP-PHAT is obtained by using the weighting function $\psi(\Omega)$ as defined in (5), which combines the increased accuracy in the SRP over the GCC and the robust weighting by the PHAT.

\section{Fourth Order Cross Cumulant}

\section{A. Kurtosis}

Kurtosis, a fourth order statistical measure, can be viewed as a measure of Gaussianity of a random process. A higher kurtosis measure corresponds to a sharper PDF compared to the Gaussian distribution. A process with sharper PDF than a Gaussian process is called super-Gaussian. Speech signals have been shown to exhibit a PDF closer to a Laplacian distribution [4], a super-Gaussian distribution, while noise sources generally exhibit a PDF closer to a Gaussian distribution.

Figure 1 shows the estimated PDF for the speech and noise signals used to evaluate the proposed method in section $\mathrm{V}$ compared to Gaussian and Laplacian noise. The PDFs are estimated using a histogram, and the signals are generated Gaussian and Laplacian noise together with recorded factory

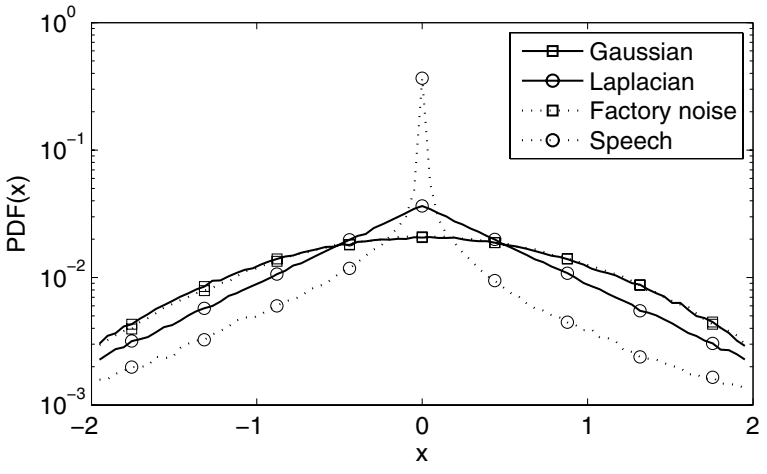

Fig. 1. PDF for Gaussian noise, Laplacian noise, factory noise and speech.

noise and speech, where all signals have a variance of unity. The PDF for factory noise is very similar to the Gaussian distribution, while the speech is closer to the Laplacian distribution.

Applications using the kurtosis measure of speech includes blind speech separation [5]. In [7] the subband kurtosis is maximized in order to extract a speech source from a noisy environment, and in [8], a modification of SRP-PHAT is presented where each subband is additionally weighted according to the subband kurtosis measure to emphasize subbands with speech present.

\section{B. Cross Cumulants}

A second order complex valued cross cumulant is the cross power spectrum [3], expressed as

$$
C_{2}\left(X, Y^{*}\right)=E\left[X Y^{*}\right]-E[X] E\left[Y^{*}\right]
$$

For zero-mean processes $X_{m}$ and $X_{n}$, the second order complex valued cross cumulant $C_{2}\left(X_{m}, X_{n}^{*}\right)$ becomes the cross power spectrum $G_{m, n}$ used as optimization target by SRP-based algorithms. A fourth order complex valued cross cumulant for two zero-mean processes $X$ and $Y$ can be expressed as

$$
C_{4}\left(X, X, Y^{*}, Y^{*}\right)=E\left[X^{2} Y^{* 2}\right]-E\left[X^{2}\right] E^{*}\left[Y^{2}\right]-2 E\left[X Y^{*}\right]^{2} .
$$

It should be noted that there are many variants of cross cumulants for complex valued random processes. For a complex valued cross cumulant of order $N$, there are $2^{N}$ ways to configure the complex conjugate on the parameters to $C_{N}$ [3], resulting in different definitions.

\section{Proposed Method}

Instead of using the SRP method, which is based on a second order complex valued cross cumulant to search for a maxima in the power response, the proposed method utilizes a fourth order complex valued cross cumulant. In subband domain, the fourth order complex valued cross cumulant used as optimization target for subband $k$ is

$$
\begin{array}{r}
K_{m, n}(k)=C_{4}\left(X_{m, k}, X_{m, k}, X_{n, k}^{*} e^{j 2 \pi \frac{k}{K} \tau}, X_{n, k}^{*} e^{j 2 \pi \frac{k}{K} \tau}\right)= \\
C_{4}\left(X_{m, k}, X_{m, k}, X_{n, k}^{*}, X_{n, k}^{*}\right) e^{j 4 \pi \frac{k}{K} \tau}
\end{array}
$$


where $X_{m, k}=X_{m, k}(t)$ is the $k$ :th subband signal for the $m:$ th sensor. The process of estimating the TDOA for a speech source, using the phase transform weighting, is therefore stated as

$$
\hat{\tau}=\arg \max _{\tau} \sum_{\{m, n\} \in P} \sum_{k=0}^{K-1} \frac{K_{m, n}(k)}{\left|K_{m, n}(k)\right|} e^{j 4 \pi \frac{k}{K} \tau}
$$

where $K$ is the number of subbands. Due to the similarities with SRP, where a cross power measure is used instead of a cross kurtosis measure, the method is herein denoted as Steered Response Kurtosis (SRK).

A DOA estimate is calculated from a TDOA estimate for a uniform linear sensor array as

$$
\hat{\alpha}=\arcsin \left(\frac{c \cdot \hat{\tau}}{f_{s} \cdot d}\right)
$$

where $c$ is the propagation speed of sound, $f_{s}$ is the sampling frequency and $d$ is the sensor spacing. The variance of the DOA estimate is [9]

$$
V[\hat{\alpha}] \propto \frac{V[\hat{\tau}]}{\cos ^{2} \alpha}
$$

where $V[\cdot]$ is the variance operator and $\alpha$ is the true DOA of the source.

\section{EVALUATION}

The proposed method for DOA estimation is evaluated and the performance is compared to the SRP-PHAT algorithm. The difference between the two methods is only the target for optimization; fourth order cross cumulant for SRK-PHAT and second order cross cumulant for the SRP-PHAT.

\section{A. Setup}

For evaluation signals, speech signals are played through a loudspeaker placed at different angles relative a 4-microphone linear sensor array with a $4 \mathrm{~cm}$ sensor spacing. Angles are defined to be 0 degrees for a source located at the broadside of the sensor array, and approaches \pm 90 degrees as the source approaches the endfire. The recordings are performed at a sample rate of $16 \mathrm{kHz}$ in a $4 \times 5 \times 2,5$ meter sized room with acoustic properties similar to a normal office room.

An oversampled uniform DFT filterbank [10] is used to transform the fullband signal into subband signals. Throughout the evaluation, a factor 2 oversampled filterbank with a prototype filter length of 4 times the number of subbands is used.

The expectation values when calculating the second and fourth order cross cumulants are estimated from a window starting $250 \mathrm{~ms}$ prior to the time of TDOA estimation.

\section{B. Speech}

The clean speech test compares SRK-PHAT and SRPPHAT for a speech signal with no added noise other than the environment noise together with reverberation created in the recording environment. A premade mask to remove estimates from periods between speech utterances is applied to both

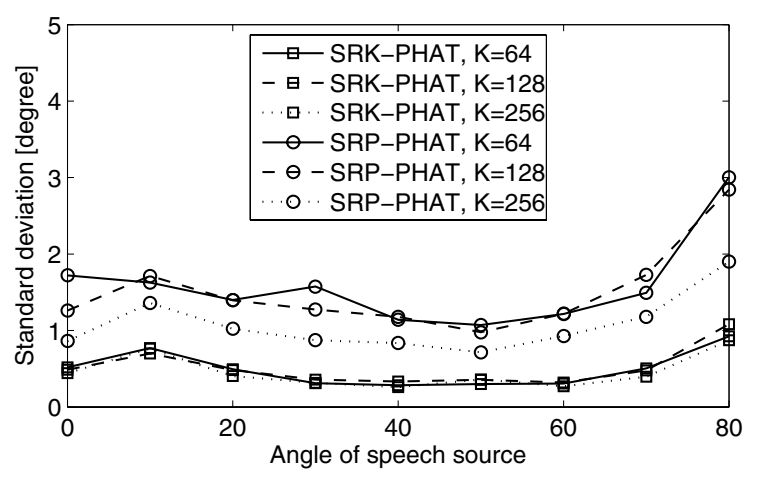

Fig. 2. Standard deviation of the DOA estimate for a clean speech source. The graphs compares SRK-PHAT and SRP-PHAT for a filterbank with 64, 128 and 256 subbands.

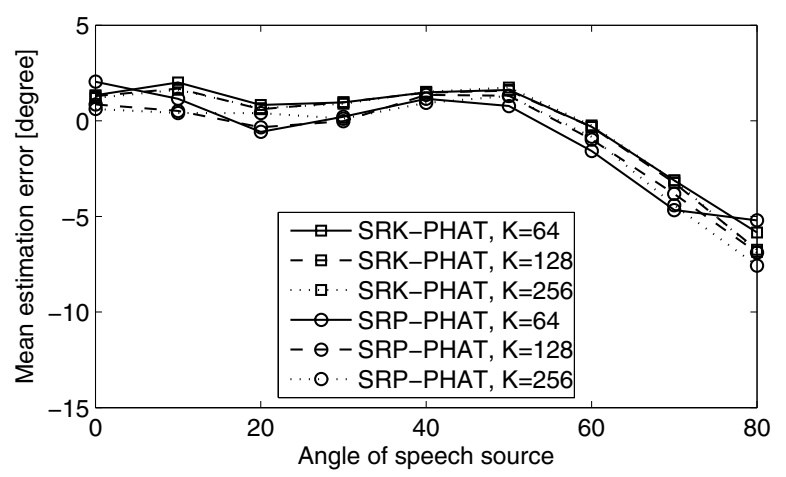

Fig. 3. Mean estimation error of the DOA estimate for a clean speech source. The graphs compares SRK-PHAT and SRP-PHAT for a filterbank with 64, 128 and 256 subbands.

methods in order to measure the statistical properties only when the speech source is active.

Figure 2 shows the standard deviation of the estimated DOA. The standard deviation is significantly less using the fourth order cross cumulant in comparison to the second order cross cumulant. From the figure it is apparent that the variance of the DOA estimates increases as the angle approaches the endfire, which is consistent with (13). Figure 3 shows the mean estimation error, which is the mean of the difference between the estimated angle and the true angle. The mean estimation errors are similar between the two methods and both seems to have a tendency to underestimate the angle as the source approaches the endfire.

As the number of subbands was decreased to 32 and below, the SRP-PHAT algorithm was not able to provide reliable DOA estimates as the variance increased tremendously, while the SRK-PHAT was able to provide DOA estimates at approximately the same variance even at 16 subbands.

\section{Speech and noise}

The same speech signals are used as in the previous test with an added noise source at different Signal to Noise Ratio (SNR). The noise is a prerecorded sequence of factory noise 


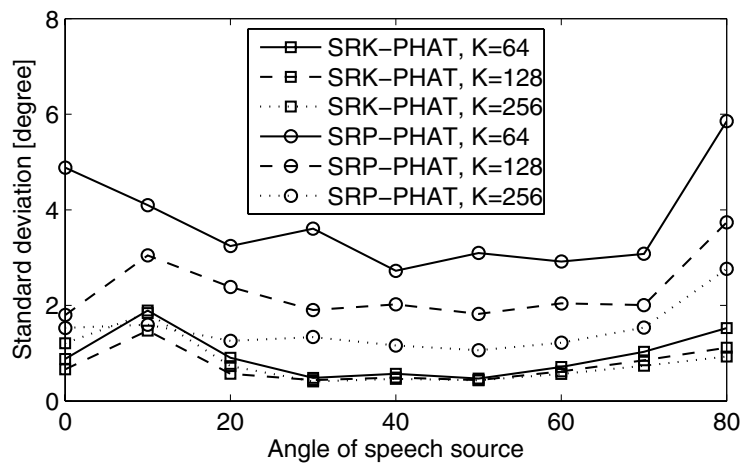

Fig. 4. Standard deviation of the DOA estimate for a speech and noise source with $0 \mathrm{~dB}$ SNR. The graphs compares SRK-PHAT and SRP-PHAT for a filterbank with 64, 128 and 256 subbands.

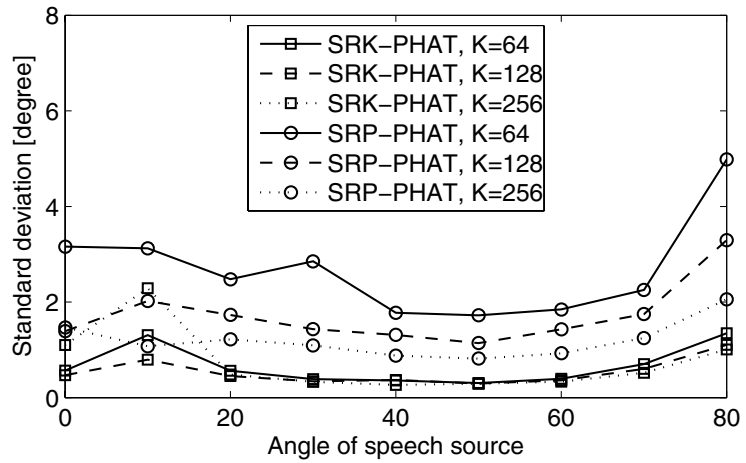

Fig. 5. Standard deviation of the DOA estimate for a speech and noise source with $10 \mathrm{~dB}$ SNR. The graphs compares SRK-PHAT and SRP-PHAT for a filterbank with 64, 128 and 256 subbands.

with machinery and moving vehicles played at an angle of -45 degrees. At time instants where speech is active, there are two sources present in the recorded signals. A DOA estimate is labeled as belonging to the speech or noise source by selecting the source with a DOA closest to the estimated DOA. Only speech DOA estimates are used in the calculation of DOA variance.

Figure 4 and 5 shows the standard deviation at SNR of 0 and $10 \mathrm{~dB}$, respectively. Also in noisy environments, the SRK-PHAT performs significantly better than SRP-PHAT.

\section{Computational complexity}

In this section, a brief analysis of computational complexity is presented. Table I shows the time in seconds spent in various parts of the DOA estimator as reported by the MATLAB profiler.

Total running time refers to the total time spent in the program. Cross cumulant estimation is the time spent estimating the fourth and second order cross cumulants. Cross cumulant optimization is the time spent in the optimizer to search for a beamformer output maxima.

The total running time is approximately doubled when fourth order cross cumulants are introduced. Optimization time
TABLE I

TIME SPENT IN PARTS OF THE SRK-PHAT AND SRP-PHAT DOA ESTIMATION ALGORITHMS.

\begin{tabular}{c||c||c}
\hline Operation & Time, SRK [s] & Time, SRP [s] \\
\hline \hline Total running time & 28 & 15 \\
\hline Cross cumulant estimation & 15 & 2 \\
\hline Cross cumulant optimization & 10 & 10 \\
\hline
\end{tabular}

is unchanged as the process of phase alteration and summing is the same. What contributes to the increased processing time is the estimation of fourth order cross cumulants over second order cross cumulants. The total running time includes subband transformation of the input signals and various one time calculations before the actual DOA estimation algorithms are executed.

\section{CONCLUSIONS}

The proposed method to use fourth order statistics over second order statistics was shown to significantly improve performance of the DOA estimator for speech sources. In all evaluated test cases regarding estimation variance, the SRK-PHAT outperforms the SRP-PHAT significantly. A brief analysis of the computational complexity was made and it shows that the complexity is approximately doubled for the whole DOA estimation procedure.

\section{REFERENCES}

[1] Z. Yermeche, N. Grbić, and I. Claesson, "Beamforming for moving source speech enhancement," in Proc. IEEE Workshop on Applications of Signal Processing to Audio and Acoustics, Oct. 2005, pp. 25-28.

[2] M. Brandstein and D. Ward, Eds., Micophone Arrays - Signal Processing Techniques and Applications. Springer, 2001.

[3] C. L. Nikias and A. P. Petropulu, Higher-Order Spectral Analysis - A Nonlinear Signal Processing Framework. Prentice-Hall, 1993.

[4] W. Zhang and S. Gazor, "Statistical modelling of speech signals," in Proc. International Conference on Signal Processing, vol. 1, Aug. 2002, pp. 480-483.

[5] J. P. LeBlanc and P. L. De Leòn, "Speech separation by kurtosis maximization," in Proc. IEEE International Conference on Acoustic, Speech and Signal Processing, vol. 2, May 1998, pp. 1029-1032.

[6] C. H. Knapp and G. C. Carter, "The generalized cross correlation method for estimation of time delay," IEEE Transactions on Acoustics, Speech, and Signal Processing, vol. ASSP-24, no. 4, pp. 320-327, Aug. 1976.

[7] B. Sällberg, N. Grbić, and I. Claesson, "Online maximization of subband kurtosis for blind adaptive beamforming in realtime speech extraction," in Proc. IEEE Digital Signal Processing, July 2007, pp. 603-606.

[8] Z. Yermeche, N. Grbić, and I. Claesson, "Blind subband beamforming with time-delay constraints for moving source speech enhancement," IEEE Transactions on Audio, Speech, and Language Processing, vol. 15, no. 8, pp. 2360-2372, Nov. 2007.

[9] S. M. Kay, Fundamentals of Statistical Signal Processing. Prentice Hall, 1993.

[10] P. P. Vaidyanathan, Multirate Systems and Filter Banks. Prentice Hall, 1993. 